Produto \& Produção, vol. 13 n. 2, p. 60-76, jun. 2012

\title{
Incerteza de medição aplicada a ensaios de indentação pós fadiga em colchões e colchonetes de espuma flexível de poliuretano
}

Recebido em 25/11/2011. Aceito em 21/05/2012.

\author{
Leandro Sosnoski \\ Universidade Federal do Rio Grande do Sul - UFRGS \\ leandro@cetemo.com.br
}

Avaliar a qualidade de colchões de espuma flexível de poliuretano condiz com o bem de consumo destinado ao repouso humano, constituído, parcial ou integralmente, por lâminas flexíveis de poliuretano, devidamente revestido. Existem normas para confecção, densidade, deformação permanente à compressão, dentre outras, para que os fabricantes e importadores de colchões mantenham a mesma qualidade dos produtos oferecidos ao consumidor. Estas normas são regidas pela ABNT Associação Brasileira de Normas Técnicas, onde esta faz referências a todos os itens normalizados que o colchão precisa ter para poder ser comercializado dentro do território nacional.

Este trabalho visa estudar a incerteza de medição no levantamento do comportamento em indentação pós fadiga da espuma para colchões de espuma flexível de poliuretano. Embora existam normas sobre a análise estatística dos resultados, avaliam-se apenas a dispersão no número de ciclos, não considerando fatores como as fontes de incerteza das tensões aplicadas, da máquina de ensaios utilizada.

Palavras-chave: indentação pós fadiga, colchão de espuma, incerteza de medição.

Assessing the quality of foam mattresses flexible polyurethane matches the consumer goods intended for human home, consisting, in part or in full by flexible polyurethane blades, properly coated. There are rules for making, density, compressive permanent deformation, among others, for manufacturers and importers of mattresses maintain the same quality of products offered to consumers. These rules are governed by ABNT - Brazilian Association of Technical Standards, where it makes references to all the standard items that the mattress need to be marketed within the territory.

This work aims to study the uncertainty of measurement in the survey of postindentation fatigue behavior of foam mattresses flexible polyurethane foam. Although there are regulations on the statistical analysis, evaluate only the dispersion in the number of cycles, not considering such factors as the sources of uncertainty of the applied voltages, the testing machine used.

Keywords: post indentation fatigue, memory foam, measurement uncertainty. 


\section{INTRODUÇÃO}

Avaliar a qualidade de colchões de espuma flexível de poliuretano condiz com o bem de consumo destinado ao repouso humano, constituído, parcial ou integralmente, por lâminas flexíveis de poliuretano, devidamente revestido conforme ABNT NBR ISO 13579-1.

O fato de se passar "metade" de nossa vida dormindo, faz com que existam normas para confecção, densidade, deformação permanente à compressão, teor de cinzas, dentre outras, para que os fabricantes e importadores de colchões mantenham a mesma qualidade dos produtos oferecidos ao consumidor. Estas normas são regidas pela ABNT - Associação Brasileira de Normas Técnicas, onde esta faz referências a todos os itens normalizados que o colchão precisa ter para poder ser comercializado dentro do território nacional.

Existem diversas lacunas no conhecimento no que diz respeito à incerteza de medição aplicada a ensaios de indentação pós fadiga em colchões e colchonetes de espuma flexível de poliuretano, apesar de existirem normas para avaliação estatística de dados e também procedimentos para estimativa da incerteza para alguns tipos de ensaio de fadiga.

A estimativa da incerteza de medição deve ser uma preocupação dos laboratórios de ensaios por ser uma das ferramentas que fornecem confiança às medições realizadas (PIZZOLATO, 2006) além de ser uma exigência para acreditação dos laboratórios de ensaios pela norma ABNT NBR ISO 17025, junto a institutos como o INMETRO, por exemplo.

As normas disponíveis sobre fadiga dizem respeito apenas a métodos para análise estatística dos dados, e não levam em consideração as incertezas advindas da máquina de ensaios utilizada, nem os que podem advir diretamente do corpo de prova. Como fonte de incerteza advinda da máquina de ensaios pode citar: a calibração dinâmica, desalinhamento, controle dinâmico de carga, inércia do sistema e aquisição de dados do sistema, vibração do sistema, etc. Para o caso do próprio corpo de prova podemos citar desalinhamento, dimensional, porosidade, etc.

Um procedimento correlato é o Uncert CoP 05 (SM\&T, 2000) que trata de ensaios de taxa de propagação de trincas. A análise estatística utilizada é a mesma utilizada para os ensaios de taxa de propagação (STEPHENS et al, 2001), onde estes dados, mesmo não sendo relevantes para espuma de poliuretano flexível, cabe o conceito para verificação de dados de máquina e do próprio corpo de prova.

Diversos trabalhos publicados recentemente tratam de alguma forma da incerteza de medição em fadiga. Geralmente tratam da incerteza de certos modelos de previsão de vida em fadiga ou da incerteza relacionada à fadiga em algumas aplicações específicas (SVENSSONS, 1997; KARADENIZ 2000; KOUTSOURELAKIS et al, 2006; BENGTSSON 2009). Existe pouco material publicado a respeito de incerteza dos resultados dos ensaios de indentação pós fadiga, que são os dados utilizados em projeto, e por isso são de grande importância. Deve ser destacado que há uma estimativa que $90 \%$ das falhas de componentes mecânicos ocorrem devido à fadiga (DIETER 1988; STEPHENS et al, 2001; CALLISTER 2007).

Existem, também, modelos mais sofisticados de redes neurais artificiais (PIERCE et al, 2008). Nestes modelos inicialmente o sistema é treinado através de dados fornecidos ao programa e depois este pode ser utilizado para prever certas soluções de diferentes problemas. Porém os resultados deste tipo de sistema 
dependerão da qualidade dos dados utilizados para fazer seu treinamento (BUCAR et al, 2006). Neste contexto também seria interessante serem conhecidos os valores de incerteza dos dados de fadiga utilizados para o treinamento do sistema, podendo estes valores ser levados em consideração ou servirem apenas como indicativos da qualidade dos dados.

Assim os objetivos deste trabalho são: listar, avaliar e quantificar as diversas fontes de incerteza envolvidas nos ensaios de indentação pós fadiga em colchões e colchonetes de espuma flexível de poliuretano; determinar as fontes mais importantes; apresentar planilhas que expressem a incerteza do ensaio.

\section{MATERIAIS E MÉTODOS}

\subsection{Fadiga Dinâmica em Colchões de Espuma Flexível de Poliuretano}

Os testes de fadiga dinâmica foram desenvolvidos para prever o desempenho ao deitar e sentar, pela aplicação de uma carga alternante, com um indentador. A fadiga é usualmente descrita como a perda de suporte de carga ou espessura em função do tempo de uso. Estudos indicam que o aumento do teor de água na formulação resulta em maior perda de suporte de carga, atribuída ao maior teor poli uréia e mais ligações de hidrogênio (VILAR, 1998). Resultados relativos ao efeito dos polióis são conflitantes. As espumas HR mostram melhor resistência à fadiga quando o peso molecular e funcionalidade do poliol aumentam, porém, a adição de dióis e trióis de baixo PM leva à redução da resistência à fadiga. A diminuição do teor de monol do poliol resulta em melhor reticulação, e aumento da resistência à tensão, ao rasgo e à fadiga. Quanto ao isocianato, o aumento do teor de isômero 2,6, leva a maior formação de poli uréia e diminuição da resistência à fadiga. Índices muito superiores a 100 podem resultar em deterioração do desempenho. Em espumas de mesma dureza, a densidade e a histerese são fatores que afetam a fadiga. Espumas com menor densidade têm pior desempenho, o que pode estar relacionado ao maior teor de poli uréia. Nas de mesma densidade a durabilidade será maior naquelas feitas com os polióis que proporcionem melhor histerese. Surfactantes que promovam uma boa estrutura celular com alta porosidade melhoram a resistência à fadiga, que geralmente piora quando a catálise da expansão e polimerização não foi bem balanceada, e com a utilização de catalisadores de estanho (VILAR, 2004).

Os fatores que contribuem para as diferenças de propriedades mecânicas entre os tipos de espuma são: a diferença na sequência das reações, que é fator determinante da morfologia do PU; o fato de que durante o crescimento das espumas de HR ocorre coalescência de algumas bolhas ocasionando uma maior dispersão de tamanho das células; e a menor abertura das células nas espumas de HR. Nas espumas HR em comparação com as convencionais, em geral o alongamento na ruptura, resistência ao rasgo, à compressão (CLD) são menores. Porém, são maiores a resiliência e o fator de conforto (próximo a três), que é expresso pela razão entre as forças de endentação a $65 \%$ e a $25 \%$. No Brasil, as propriedades de espumas flexíveis para colchões, são especificadas pela NBR 13579-1 (Tabela 2.1). 
Tabela 2.1 - Propriedade das espumas segundo NBR 13579-1

\begin{tabular}{|c|c|c|c|c|c|c|c|c|}
\hline Propriedades & $\mathrm{D} 18^{\star \star \star}$ & D20 & D23 & D26 & D28 & D33 & D40 & D45 \\
\hline $\begin{array}{l}\text { Força de indentação (FI) - Dureza* a } \\
40 \%, N \text { (min) }\end{array}$ & 80 & 95 & 110 & 130 & 145 & 165 & 185 & 200 \\
\hline Densidade $\left(\mathrm{kg} / \mathrm{m}^{3}\right),(\mathrm{min})$ & 16,2 & 18,0 & 20,7 & 23,4 & 25,2 & 29,7 & 36,0 & 40,5 \\
\hline Tensão de ruptura $(\mathrm{kPa}),(\mathrm{min})$ & 70 & 90 & 90 & 90 & 90 & 90 & 90 & 90 \\
\hline Alongamento \%, (min) & 120 & 120 & 120 & 120 & 120 & 120 & 100 & 100 \\
\hline Resistência ao rasgo (N/m), (min) & 400 & 450 & 450 & 450 & 450 & 450 & 450 & 450 \\
\hline Resiliência \% (min) & \multicolumn{2}{|c|}{30} & \multicolumn{4}{|c|}{35} & \multicolumn{2}{|c|}{40} \\
\hline \begin{tabular}{|lll} 
Deformação & permanente \\
compressão & $90 \%, \%$ (máx)
\end{tabular} & 12 & \multicolumn{3}{|c|}{10} & \multicolumn{4}{|c|}{8} \\
\hline Suporte de carga, $65 \%(\mathrm{~N}),(\mathrm{min})$ & 140 & 160 & 180 & 220 & 240 & 270 & 330 & 340 \\
\hline Fator de conforto ${ }^{* *},(\mathrm{~min})$ & \multicolumn{2}{|c|}{2,0} & 2 & 1 & \multicolumn{4}{|c|}{2,2} \\
\hline Cinzas \%, (máx) & \multicolumn{8}{|c|}{1} \\
\hline \begin{tabular}{|llll} 
Fadiga dinâmica & - & Perda & de \\
espessura $\%,($ máx $)$ & & & \\
\end{tabular} & 8 & 6 & \multicolumn{3}{|c|}{5} & \multicolumn{3}{|c|}{4} \\
\hline Perda de suporte, $25 \%$, (máx) & 30 & 30 & 25 & 25 & 25 & 25 & 25 & 25 \\
\hline $\begin{array}{l}\text { * A força de indentação pode ser exp } \\
\text { fadigada. } \\
\star * \text { O fator de conforto pode ser expre }\end{array}$ & & & & & & & & \\
\hline
\end{tabular}

\subsection{Incerteza de Medição}

A incerteza de medição é um parâmetro não-negativo que caracteriza a dispersão de um valor quantitativo atribuído a um mensurando (grandeza submetida à medição) baseado nas informações usadas (JCGM, 2008b traduzido pelo autor).

O cálculo de incerteza de medição faz uso de cálculos estatísticos, mas é diferente de uma simples análise estatística baseada em média e desvio padrão. Pois esta além de envolver vários passos de análise também leva em consideração outras fontes de incerteza. Vejamos um exemplo: se for realizado diversos ensaios de tração para a determinação da tensão de ruptura de certo material, pode-se analisar os resultados estatisticamente a partir da média e do desvio padrão dos valores obtidos. Assim estar-se-ia analisar a partir do desvio padrão a dispersão dos resultados. Já no cálculo da incerteza de medição além dessa dispersão nos resultados pode-se ainda levar em conta a incerteza da carga aplicada e também das medidas utilizadas para calcular a área dos corpos de prova, valores esses que podem ser obtidos, por exemplo, dos certificados de calibração dos equipamentos utilizados.

Passos para determinação da incerteza de medição (EA-4/02):

1. Definir o mensurando e seu modelo;

2. Identificar as fontes de incerteza;

3. Quantificar as fontes de incerteza;

4. Calcular os coeficientes de sensibilidade;

5. Calcular as componentes de incerteza;

6. Calcular a incerteza combinada;

7. Calcular o número de graus de liberdade efetivos; 
8. Calcular o coeficiente de abrangência;

9. Calcular a incerteza expandida.

Em geral o mensurando não é medido diretamente, mas sim determinado em função de outras grandezas. Sendo o mensurando $Z$ determinado a partir das grandezas $X_{1}, X_{2}, X_{3}, \ldots, X_{L}$, através da Equação 2.1, a incerteza de medição combinada pode ser determinada a partir da Equação 2.2. No caso das grandezas $X_{1}, X_{2}, X_{3}, \ldots, X_{L}$, serem independentes a Equação 2.2 pode ser simplificada na Equação 2.3 (JCGM. 2008a adaptada pelo autor).

$$
\begin{array}{cr}
Z=f\left(X_{1}, X_{2}, X_{3}, \ldots, X_{L}\right) & \text { Equação } \\
u_{0_{Z}}^{2}=\sum_{i=1}^{L}\left(\frac{\partial f}{\partial x_{i}}\right)^{2} u_{x_{i}}^{2}+2 \sum_{i=1}^{L} \sum_{j=i+1}^{L}\left(\frac{\partial f}{\partial x_{i}}\right) \cdot\left(\frac{\partial f}{\partial x_{j}}\right) u_{x_{i}} \cdot u_{x_{j}} \cdot r\left(x_{i}, x_{j}\right) & \text { Equação } \\
u_{O_{Z}}^{2}=\sum_{i=1}^{L}\left(\frac{\partial f}{\partial x_{i}}\right)^{2} u_{x_{i}}^{2} & \text { Equação } \\
\end{array}
$$

As derivadas parciais presentes na expressão são denominadas coeficientes de sensibilidade $\left(c_{x i}\right)$. E o produto do coeficiente de sensibilidade de uma grandeza e sua incerteza é denominado componente de incerteza. A partir da análise dos componentes de incerteza pode-se verificar quais grandezas que mais contribuem para a incerteza na medição do mensurando $Z$ e, se necessário, tentar reduzir sua influência.

O cálculo do número de graus de liberdade efetivos $\left(v_{e f f}\right)$ pode ser feito através da Equação 2.4. Com o valor de $v_{e f f}$ e com o nível de confiança (geralmente 95\%) é determinado o coeficiente de abrangência com o uso de tabelas da distribuição $t$-Student. E, finalmente, a incerteza expandida é obtida conforme Equação 2.5.

$$
\begin{gathered}
v_{e f f}=\frac{u_{Z}^{4}}{\sum \frac{u_{x_{j}}^{4}}{v_{x_{i}}}} \\
U_{Z}=k \cdot u_{0_{z}}
\end{gathered}
$$

Tendo obtido um valor para $v_{\text {eff }}$, a tabela $t$-Student será utilizada para encontrar um valor de $t_{p}(v)$. A Tabela 2.2 apresenta alguns valores para $t_{95,45}(v)$.

Tabela 2.2 - t-Student segundo EA-4/02

\begin{tabular}{|c|c|c|c|c|c|c|c|c|c|c|c|c|}
\hline$v_{\text {eff }}$ & 1 & 2 & 3 & 4 & 5 & 6 & 7 & 8 & 10 & 12 & 14 & 16 \\
\hline$t_{95,45}(v)$ & 13,9 & 4,53 & 3,31 & 2,87 & 2,65 & 2,52 & 2,43 & 2,37 & 2,28 & 2,23 & 2,20 & 2,17 \\
\hline
\end{tabular}




\begin{tabular}{|c|c|c|c|c|c|c|c|c|c|c|c|c|}
\hline$v_{\text {eff }}$ & 18 & 20 & 25 & 30 & 35 & 40 & 45 & 50 & 60 & 80 & 100 & $\infty$ \\
\hline$t_{95,45}(v)$ & 2,15 & 2,13 & 2,11 & 2,09 & 2,07 & 2,06 & 2,06 & 2,05 & 2,04 & 2,03 & 2,02 & 2,00 \\
\hline
\end{tabular}

As incertezas padrão individuais são combinadas para produzir um valor total de incerteza, denominada como incerteza padrão combinada. Esta incerteza padrão combinada da estimativa y é representada na Equação 2.6. A incerteza padronizada é dada pela Equação 2.7 .

$$
\begin{array}{cr}
u_{c}=\sqrt{a_{1}^{2} \cdot c_{s 1}+a_{2}^{2} \cdot c_{s 2}+\ldots+a_{n}^{2} \cdot c_{s n}} & \text { Equação } \\
& 2.6 \\
u_{p}=\frac{U_{z}}{k} & \text { Equação } \\
& 2.7
\end{array}
$$

\subsubsection{Tipo de Fonte de Incerteza} 2008a).

As fontes de incerteza podem ser divididas em dois tipos: A e B (JCGM,

Incertezas do tipo A: são provenientes de observações repetidas, nesse caso o valor da incerteza (u) pode ser estimado como o desvio padrão (s) dividido pela raiz do número de observações realizadas.

A dispersão dos resultados indica a repetitividade do processo de medição e depende dos equipamentos utilizados, do método, e algumas vezes do técnico que está efetuando a medição. A função estatística utilizada para caracterizar a dispersão nos resultados é o desvio padrão amostral dos $\mathrm{n}$ valores que compõe a amostra, conforme Equação 2.8.

$$
s\left(x_{k}\right)=\sqrt{\frac{1}{(n-1)} \sum_{k=1}^{n}\left(x_{k}-\bar{x}\right)^{2}}
$$

Equação

Neste caso, a incerteza padrão será caracterizada pelo desvio padrão experimental da média, dado pela Equação 2.9.

$$
s(\bar{x})=\frac{s}{\sqrt{n}}
$$

Incertezas do tipo B: são aqueles pelo julgamento, conhecimentos prévios dos equipamentos, certificados, manuais, etc. Os casos mais comuns deste tipo de incerteza são listados na Tabela 2.3, sendo "a" um valor estimado/parâmetro das distribuições. A distribuição uniforme geralmente é usada quando se conhecem os valores máximo e mínimo de erro de uma medida e não se tem informação alguma sobre sua distribuição. A distribuição triangular é usada quando se conhecem os valores máximo e mínimo e também é conhecido o valor mais provável.

Algumas vezes a mesma fonte de incerteza pode ser considerada do tipo $\mathrm{A}$ ou $\mathrm{B}$ dependendo da forma pela qual ela é estimada. 
Tabela 2.3 - Incertezas tipo B segundo JCGM 2008a

\begin{tabular}{|c|c|c|}
\cline { 2 - 3 } \multicolumn{1}{c|}{} & Valor de incerteza & Gráfico da distribuição \\
\hline Distribuição Uniforme ou Retangular & $u=\frac{a}{\sqrt{3}}$ & \\
\hline $\begin{array}{c}\text { Distribuição Triangular com uso } \\
\text { de instrumento com indicação digital }\end{array}$ & $u=\frac{a}{\sqrt{6}}$ & \\
\hline Certificado & $u=\frac{r}{2 \sqrt{3}}$ & \\
\hline & $u=\frac{U}{k}$ & \\
\hline
\end{tabular}

onde, $r$ = resolução do instrumento a ser calibrado.

\subsubsection{Importância da Incerteza de Medição}

Sendo o resultado de uma medição apenas uma estimativa do valor verdadeiro, a incerteza é necessária para expressar o grau de dúvida associado a este resultado. Assim é fácil notar que a incerteza é um dos instrumentos que fornece confiança às medições. Os outros instrumentos que fornecem confiança nas medidas são listados abaixo (PIZZOLATO, 2006):

- Sistema Internacional de Unidade (SI)

- Rastreabilidade

- Materiais de referência

- Acordos de reconhecimento mútuo

- Ensaios de proficiência

- Acreditação

A incerteza também é fundamental em situações como interpretações de resultados de medições e para verificação de conformidade tanto na área de ensaios quanto na de calibração (EA-4/16. 2003).

A incerteza também pode ser um diferencial competitivo, pois o cliente tende a buscar o laboratório com melhor qualidade em suas medidas e, portanto, a menor incerteza. Adicionalmente, a incerteza é uma ferramenta valiosa para o laboratório, pois permite a identificação dos fatores que mais influenciam no resultado da medição. Desta forma é possível programar formas de controle adequadas para a garantia da qualidade e da melhoria contínua (JORNADA, 2009). 


\subsection{Diagrama de Realização de Ensaios}

Para a realização dos ensaios são necessários alguns procedimentos, (ABNT NBR ISO 9176 e 9177) conforme demonstrado no diagrama a seguir:

Medição das Dimensões do Colchão

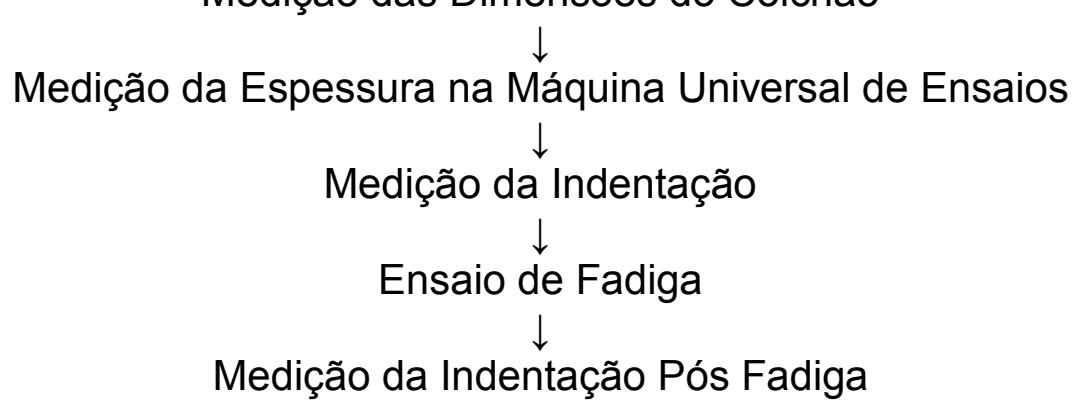

\subsection{Material e Corpos de Prova}

Para entender dos materiais e corpos de prova que serão usados nos ensaios deve-se descrever o seguinte:

COLCHÃO DE ESPUMA FLEXÍVEL DE POLIURETANO: Bem de consumo destinado ao repouso humano, constituído, parcial ou integralmente, por lâmina(s) flexível(eis) de poliuretano, devidamente revestido.

Densidade nominal (D): valor numérico que indica a densidade de referência expressa em $\mathrm{kg} / \mathrm{m}^{3}$;

Densidade real (Dr): valor numérico obtido por metodologia de ensaio, expressa em $\mathrm{kg} / \mathrm{m}^{3}$;

Fator de conforto (F.C): relação entre a força de indentação a $65 \%$ e a força de indentação a $25 \%$.

\subsubsection{Dimensões do Colchão para Ensaio}

As dimensões totais da espessura, largura e comprimento, assim como da espessura da lâmina de espuma devem ser verificadas da seguinte forma:

Medição do comprimento, largura e altura do colchão e colchonete acabado:

a) Estender o colchão/colchonete sobre uma superfície plana e rígida maior que a área do colchão/colchonete;

b) Manter em repouso o colchão/colchonete por um período não inferior a 30 (trinta) minutos;

c) Medir a largura total com a trena, sendo considerado o revestimento, colocar os dois esquadros sobre a superfície plana, encostando as faces verticais $\left(90^{\circ}\right)$ nas laterais do colchão/colchonete, em posições opostas, em três pontos equidistantes, sendo considerado como sua largura total o valor médio encontrado, expresso em centímetros;

d) Medir o comprimento total com a trena, sendo considerado o revestimento, colocar os dois esquadros sobre a superfície plana, encostando as faces verticais $\left(90^{\circ}\right)$ nas extremidades do colchão/colchonete, em posições opostas, em três pontos 
equidistantes, considerado como seu comprimento total o valor médio encontrado, expresso em centímetros;

e) Medir a altura total, sendo considerado o revestimento, com a régua ou escala milimétrica. Apoiar longitudinalmente no centro do colchão/colchonete, uma régua sem escala, de forma a ultrapassar as extremidades, a distância entre a superfície de apoio do colchão/colchonete e a parte inferior da régua nas duas extremidades, considerando como sua altura total o valor médio encontrado, expresso em centímetros.

Medição da espessura da lâmina de espuma do colchão/colchonete:

a) Após retirar o revestimento, estender a(s) lâmina(s) de espuma do colchão/colchonete sobre uma superfície plana e rígida maior que a área da lâmina;

b) Manter em repouso a(s) lâmina(s) de espuma por um período não inferior a 30 (trinta) minutos;

c) Medir a espessura da(s) lâmina(s) de espuma utilizada na(s) face(s) do colchão/colchonete, com a régua ou escala milimétrica. Apoiar longitudinalmente no centro da lâmina de espuma, uma régua sem escala, de forma a ultrapassar as extremidades, a distância entre a superfície de apoio da lâmina de espuma e a parte inferior da régua nas duas extremidades, considerando como sua espessura o valor médio encontrado, expresso em centímetros.

Nota: No caso de produtos que apresentarem mais de uma lâmina de espuma deve-se executar o procedimento acima em todas as lâminas, não sendo necessário o descolamento entre as lâminas.

- São admitidas tolerâncias com relação à largura e comprimento do produto acabado de $\pm 1,5 \mathrm{~cm}$ e para a altura de $-0,5 /+1,5 \mathrm{~cm}$, com base nas dimensões declaradas na etiqueta pelo fabricante.

- A espessura mínima da lâmina do colchão ou colchonete, sem o revestimento, deve corresponder à medida especificada na Tabela 2.4, sendo admitida tolerância de $\pm 0,5 \mathrm{~cm}$, da espessura total e individual de cada lâmina. A espessura é referente à lâmina de espuma sem revestimento.

- A soma das espessuras dos materiais que compõem o revestimento do colchão (infantil, geral, misto) não deve exceder $1 / 3$ da altura total;

* Colchonete: a altura total não pode exceder $8 \mathrm{~cm}$, inclusive com 0 revestimento;

* Conjugado e auxiliar: A soma das espessuras dos materiais que compõem o revestimento do colchão conjugado e auxiliar não deve exceder $1 / 3$ da espessura da lâmina de espuma.

Tabela 2.4 - Dimensões mínimas: colchonete e colchão de espuma flexível e poliuretano segundo NBR 13579-1

\begin{tabular}{|l|c|c|}
\hline \multirow{2}{*}{\multicolumn{1}{|c|}{ Tipos }} & \multicolumn{2}{c|}{ Espessuras } \\
\cline { 2 - 3 } & Mínima $(\mathrm{cm})$ & Máxima $(\mathrm{cm})$ \\
\hline Colchonete $^{\text {a) }}$ & 4 & Não há \\
\hline Colchão infantil $^{*}$ & 7 & Não há \\
\hline Colchão geral $^{*}$ & 12 & Não há \\
\hline Colchão auxiliar $^{\text {b) }}$ & 5 & Não há \\
\hline Colchão box conjugado $^{\text {b) }}$ & 5 & Não há \\
\hline Colchão misto $^{\text {b) }}$ & 5 & \\
\hline
\end{tabular}


a) Colchonete nas medidas acabadas, com revestimento;

b) Colchão auxiliar, box conjugado e misto deve ter densidade $\geq 28$.

\subsubsection{Metodologia e Detalhamento}

São necessários para cada ensaio um mínimo de 04 corpos de prova por amostra, sendo que 03 serão selecionados para o ensaio e 01 servirá como testemunha.

Verificar as dimensões dos corpos de prova que devem ser de no mínimo $(380 \pm 10) \times(380 \pm 10) \times(50 \pm 2) \mathrm{mm}$, tendo suas bases planas e paralelas e as faces laterais perpendiculares às mesmas. Os corpos de prova podem ser obtidos pelo empilhamento de no máximo duas camadas do mesmo material, sem a utilização de adesivo.

Preparar os seguintes equipamentos e instrumentos:

1) Deve-se preparar a Máquina Universal de Ensaio de 30 toneladas, com célula de carga de $500 \mathrm{kgf}$ e dispositivos para a execução do ensaio conforme Figura 1.

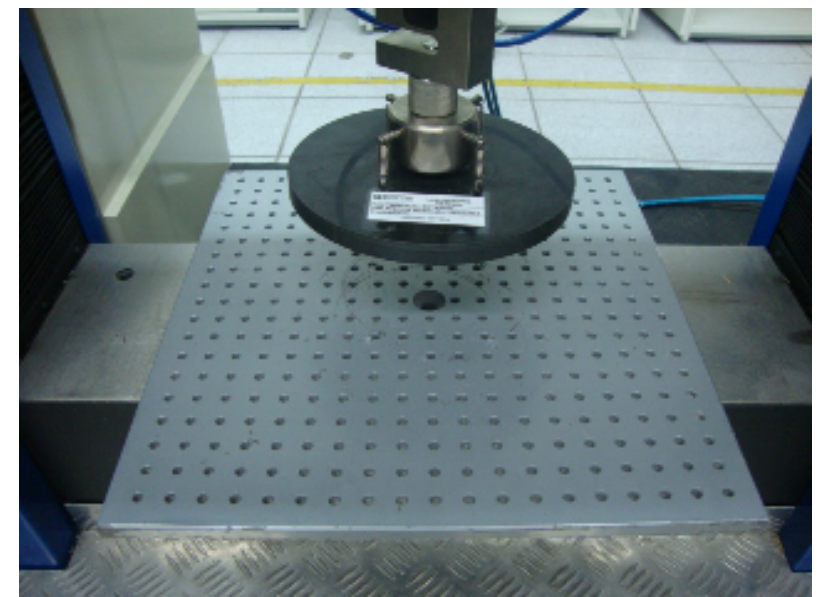

Figura 1 - Montagem da placa de apoio e indentador da máquina Universal de Ensaio.

2) Máquina de Fadiga em espuma, capaz de produzir um movimento uniforme de compressão e descompressão da espuma com uma frequência de $(70 \pm 10)$ ciclos $/ \mathrm{min}$.

3) Montar a placa de apoio na Máquina Universal de Ensaios consiste em colocar uma chapa metálica rígida horizontal, contendo furos de $(6,5 \pm 0,5) \mathrm{mm}$, distanciados de aproximadamente $(20 \pm 1) \mathrm{mm}$ um do outro, a fim de permitir escape do ar durante o ensaio.

4) $O$ indentador metálico deve ser rígido, com diâmetro de $(250 \pm 10) \mathrm{mm}$, borda arredondada de raio $(25 \pm 5) \mathrm{mm}$, com um dispositivo que permita comprimir a espuma flexível em $(70 \pm 5) \%$ e uma espuma aglomerada em $(50 \pm 5) \%$ da espessura inicial. $O$ indentador deve ser fixado rigidamente a sua guia e sua superfície deve ser lisa, mas não polida. 


\section{EXECUÇÃO:}

As amostras utilizadas são feitas de espuma flexível de poliuretano com densidade pré-estabelecida pelo fabricante, modelo D-33.

Antes da realização do ensaio e somente após $24 \mathrm{~h}$ de sua manufatura, os corpos de prova foram condicionados de 12 horas a temperatura de $(23 \pm 2)^{\circ} \mathrm{C}$ e $(50 \pm 5) \%$ de umidade relativa, onde esta condição foi monitorada.

Foram ensaiados três corpos de prova para cada amostra, onde a realização do ensaio foi na direção (ou lado) na qual o produto final será solicitado.

$\mathrm{Na}$ máquina universal de ensaios, realizou-se o ensaio de indentação da seguinte forma:

- Por três vezes seguidas, o corpo de prova foi pré-comprimido a $(70 \pm 5) \%$ da espessura original a uma velocidade de $(100 \pm 10) \mathrm{mm} / \mathrm{min}$. Aliviou-se a força após atingir os $(70 \pm 5) \%$ de compressão na mesma velocidade.

- Comprimiu-se o corpo de prova a $(25 \pm 1) \%$ de sua espessura original. Manteve-se esta compressão por $(30 \pm 1)$ s. Mediu-se então a força de indentação a $(25 \pm 1) \%$.

- Aumentou-se a compressão do corpo de prova para $(40 \pm 1) \%$ de sua espessura original. Manteve-se esta compressão por $(30 \pm 1)$ s. Mediu-se então a força de indentação a $(40 \pm 1) \%$.

- Aumentou-se a compressão do corpo de prova para $(65 \pm 1) \%$ de sua espessura original. Manteve-se esta compressão por $(30 \pm 1)$ s. Mediu-se então a força de indentação a $(65 \pm 1) \%$.

Após as compressões terem sido feitas imprimiu-se o relatório fornecido pelo software da máquina.

Foram colocados os corpos de prova na máquina de fadiga em espuma sobre a placa de apoio, de modo que ficaram centralizados sob o indentador. Ajustou-se a amplitude do ciclo para que a espuma flexível seja comprimida a $(70 \pm 5) \%$.

Ajustou-se a máquina de fadiga para uma espuma com $50 \mathrm{~mm}$ de espessura (espuma flexível - 70\%), deve-se reduzir até $15 \mathrm{~mm}$ de sua espessura. Pode-se ver o ajuste da máquina conforme mostra a Figura 2.

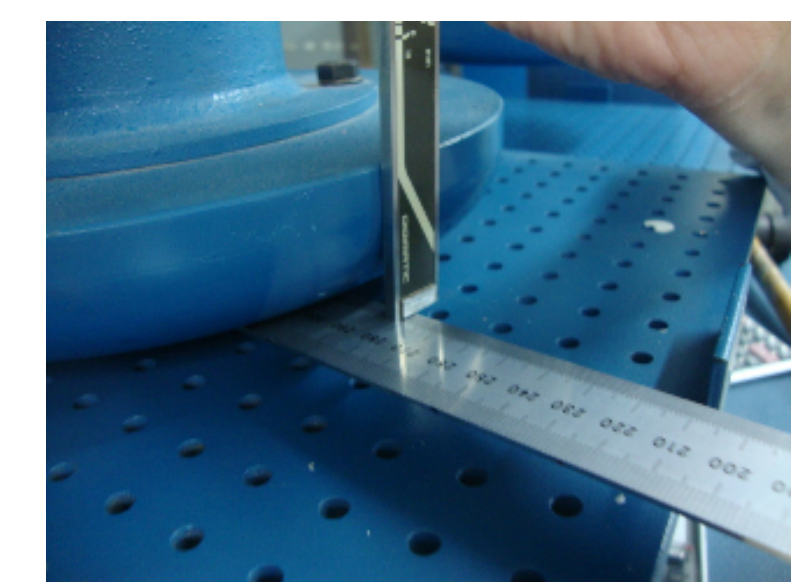

Figura 2 - Verificação da amplitude do ciclo na Máquina de Fadiga.

Foram realizados 80.000 ciclos contínuos (a máquina leva em torno de 19 horas para finalizar o ensaio) e após retirou-se o corpo de prova do equipamento, deixando-o em repouso por 20 minutos. 
Após, na máquina universal de ensaios, determinar a espessura $\left(E_{f}\right)$ e a força de indentação $\left(\mathrm{FI}_{\mathrm{f}}\right)$, excluindo-se a pré-compressão a $(70 \pm 5) \%$, onde foi executado o ensaio de pós fadiga (conforme NBR 9177), que se repete com relação ao ensaio de indentação anterior.

\section{EXPRESSÃO DOS RESULTADOS:}

O resultado é a média aritmética dos três ensaios efetuados para cada compressão (25\%, 40\% e 65\%), expresso como força de indentação, em Newton, com precisão de $1 \mathrm{~N}$. ASTM 3574):

Calcular o fator de conforto utilizando a equação abaixo (baseada na

$$
\text { Fator de conforto }=\frac{\text { força de indentação a } 65 \%}{\text { força de indentação a } 25 \%}
$$

Perda de Espessura:

A perda de espessura é calculada pela equação:

$$
P_{E}=\frac{E_{0}-E_{f}}{E_{f}} .100
$$

Onde:

$\mathbf{P}_{\mathrm{E}}$ é a perda em espessura, em porcentagem;

$E_{0}$ é a espessura original, em milímetros;

$\mathbf{E}_{\mathrm{f}}$ é a espessura final, em milímetros.

Perda de Força de Indentação:

A perda de força de indentação é calculada pela equação:

$$
P_{F I}=\frac{F I_{0}-F I_{f}}{F I_{0}} .100
$$

Onde:

$\mathbf{P}_{\mathbf{F I}}$ é a perda de força de indentação percentual, em porcentagem;

$\mathrm{FI}_{0}$ é a força de indentação a 25\%, 40\% e 65\% antes do ensaio, em Newton; Newton.

$\mathrm{Fl}_{\mathbf{f}}$ é a força de indentação a $25 \%, 40 \%$ e $65 \%$ após o ensaio de fadiga, em

O relatório de ensaio deve conter a perda de espessura e a perda de força de indentação.

\section{RESULTADOS E DISCUSSÕES}

Os resultados obtidos no ensaio de indentação foram obtidos na máquina bem como o cálculo do fator de conforto conforme Tabela 3.1. A Figura 3 mostra o gráfico Força $(\mathrm{N}) \times$ Deformação Específica (\%), para indentação dos corpos de prova.

Tabela 3.1 - Resultados do ensaio de indentação e cálculo do fator de conforto.

\begin{tabular}{|l|l|l|l|l|l|}
\hline Corpo de & Espessura & Força de & Força de & Força de & Fator de \\
\hline
\end{tabular}




\begin{tabular}{|l|c|c|c|c|c|}
\hline \multicolumn{1}{|c|}{ prova } & $\begin{array}{c}\text { original } \\
(\mathrm{mm})\end{array}$ & $\begin{array}{c}\text { indentação a } \\
25 \%(\mathrm{~N})\end{array}$ & $\begin{array}{c}\text { indentação a } \\
40 \%(\mathrm{~N})\end{array}$ & $\begin{array}{c}\text { indentação a } \\
65 \%(\mathrm{~N})\end{array}$ & conforto \\
\hline CP1 & 53,4 & 145,4 & 186,6 & 357,7 & 2,46 \\
\hline $\mathrm{CP} 2$ & 51,1 & 142,8 & 182,3 & 355,5 & 2,49 \\
\hline $\mathrm{CP} 3$ & 50,8 & 146,1 & 184,4 & 347,1 & 2,38 \\
\hline $\begin{array}{l}\text { Número } \\
\text { de CPs }\end{array}$ & 3 & 3 & 3 & 3 & 3 \\
\hline Média & 51,79 & 144,8 & 184,4 & 353,4 & 2,441 \\
\hline $\begin{array}{l}\text { Desvio } \\
\text { Padrão }\end{array}$ & 1,419 & 1,734 & 2,165 & 5,563 & 0,05863 \\
\hline $\begin{array}{l}\text { Coef. } \\
\text { Var. (\%) }\end{array}$ & 2,741 & 1,189 & 1,174 & 1,574 & 2,402 \\
\hline Mínimo & 50,83 & 142,8 & 182,3 & 347,1 & 2,376 \\
\hline Máximo & 53,42 & 146,1 & 186,6 & 357,7 & 2,488 \\
\hline
\end{tabular}

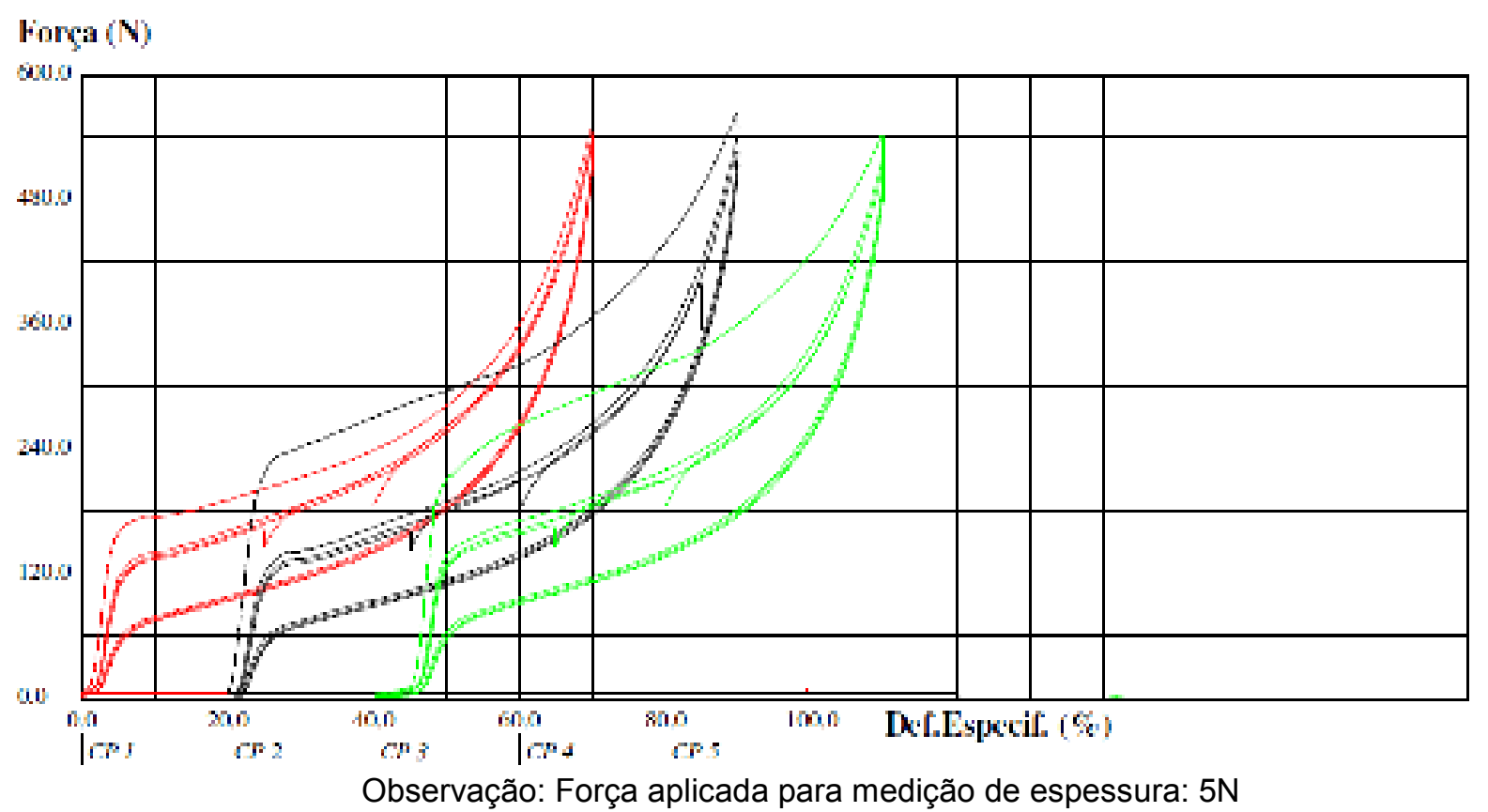

Figura 3 - Gráfico Força (N) X Deformação Específica (\%) para indentação.

Na Tabela 3.2 e Tabela 3.3 estão determinadas às forças de indentação, fator de conforto, perda de espessura e perda de força de indentação conforme pede a norma NBR 9176 e NBR 9177. O cálculo de perda de espessura utiliza a Equação 4.2 e a perda de força de indentação a Equação 4.3.

Tabela 3.2 - Determinação da força de indentação e fator de conforto. 


\begin{tabular}{|c|c|c|c|c|}
\hline Amostra & $\begin{array}{c}\text { Força de } \\
\text { indentação a } 25 \% \\
(\mathrm{~N})\end{array}$ & $\begin{array}{c}\text { Força de } \\
\text { indentação a } 40 \% \\
(\mathrm{~N})\end{array}$ & $\begin{array}{c}\text { Força de } \\
\text { indentação a } 65 \% \\
(\mathrm{~N})\end{array}$ & $\begin{array}{c}\text { Fator de } \\
\text { conforto } \\
\text { médio* }\end{array}$ \\
\hline 01 & 144,8 & 184,4 & 353,4 & 2,44 \\
\hline
\end{tabular}

*Para cada corpo de prova, utiliza-se a Equação 2.10.

Tabela 3.3 - Determinação da perda de espessura e da perda de força de indentação (Fadiga Dinâmica).

\begin{tabular}{|c|c|c|c|c|}
\hline Amostra & $\begin{array}{c}\text { Perda de força de } \\
\text { indentação a 25\% } \\
(\%)\end{array}$ & $\begin{array}{c}\text { Perda de força de } \\
\text { indentação a 40\% } \\
(\%)\end{array}$ & $\begin{array}{c}\text { Perda de força de } \\
\text { indentação a 65\% } \\
(\%)\end{array}$ & $\begin{array}{c}\text { Perda de } \\
\text { espessura } \\
(\%)\end{array}$ \\
\hline 01 & 15,44 & 19,17 & 18,33 & 1,65 \\
\hline
\end{tabular}

$\mathrm{Na}$ Tabela 3.4, encontra-se a planilha da incerteza do ensaio de indentação para uma célula de carga de $500 \mathrm{kgf}$.

Tabela 3.4 - Incerteza do ensaio de indentação para célula de carga de 500kgf.

\begin{tabular}{|c|c|c|c|c|c|c|}
\hline $\begin{array}{l}\text { FONTE DE } \\
\text { INCERTEZA }\end{array}$ & $\begin{array}{c}\text { Valor } \\
\text { Incerteza } \\
\text { Expandid } \\
\text { a }(\mathrm{N})\end{array}$ & $\begin{array}{c}\text { Distribuição } \\
\text { de } \\
\text { Probabilidade }\end{array}$ & Divisor & $\begin{array}{l}\text { Coeficiente } \\
\text { Sensibilidad } \\
\text { e (Cs) }\end{array}$ & $\begin{array}{c}\text { Incerteza } \\
\text { Padronizad } \\
\text { a (N) }\end{array}$ & $\begin{array}{l}\text { Graus de } \\
\text { Liberdade }\end{array}$ \\
\hline $\begin{array}{l}\text { Erro de divisão } \\
\text { de escala } \\
\text { (Máquina } \\
\text { Universal) }\end{array}$ & 0,0500 & Retangular & 1,73 & 1 & 0,029 & Infinitos \\
\hline $\begin{array}{l}\text { Incerteza na } \\
\text { calibração da } \\
\text { célula na } \\
\text { compressão }\end{array}$ & 1,9000 & Retangular & 1,73 & 1 & 1,097 & Infinitos \\
\hline $\begin{array}{l}\text { Maior erro da } \\
\text { escala na } \\
\text { compressão }\end{array}$ & 5,0000 & Retangular & 1,73 & 1 & 2,887 & Infinitos \\
\hline $\begin{array}{l}\text { Desvio padrão } \\
\text { da amostra } \\
\text { (Divisor } t- \\
\text { Student } 2 \text { pois } \\
\text { são } 3 \text { corpos de } \\
\text { prova logo } n-1= \\
\text { 2) }\end{array}$ & 1,4190 & Normal & 4,53 & 1 & 0,313 & 2 \\
\hline \multicolumn{5}{|c|}{ Incerteza padronizada combinada (N) } & 3,104 & \\
\hline \multicolumn{3}{|c|}{ Incerteza expandida $(\mathrm{N})$} & $k=$ & 2 & 6,208 & $\begin{array}{c}267394233, \\
6 \\
\end{array}$ \\
\hline
\end{tabular}


As fontes de incerteza no ensaio de indentação foram consideradas conforme o diagrama Ishikawa apresentado abaixo.

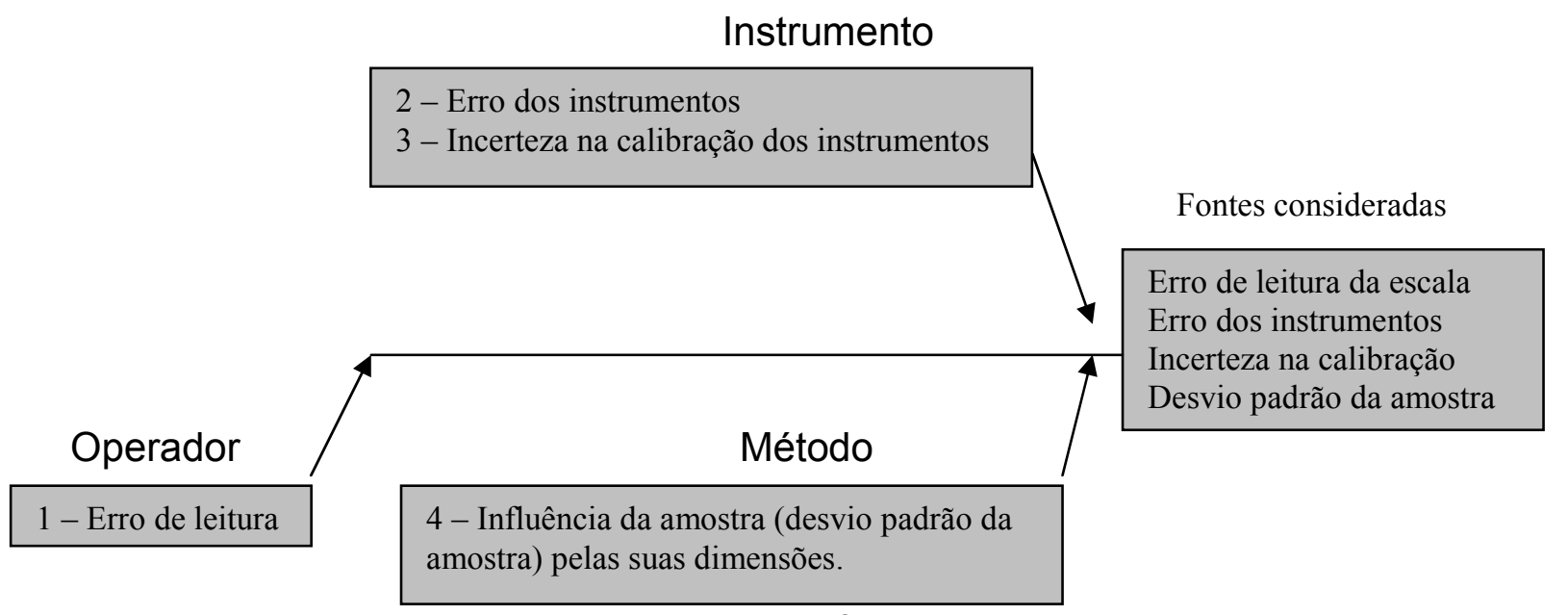

Figura 4 - Diagrama Ishikawa para fontes de incerteza de indentação.

Percebe-se nas planilhas e gráficos que os dados ficaram dentro do estabelecido pelas normas, isso indica que os corpos de prova ensaiados ficaram dentro do esperado, fazendo assim que eles sejam liberados para fabricação e colocação dos produtos no comércio.

Outro ponto de discussão é relacionado com o número de corpos de prova exigidos pela norma, no caso três, para que se obtenha um melhor coeficiente de $t$ student, o número de corpos de prova deveria ser aumentado significativamente para que os valores obtidos nos cálculos de incerteza ficassem menores.

\section{CONCLUSÃO}

Com base na Tabela 3.4, pode ser verificar que a incerteza padronizada combinada foi de aproximadamente $3 \mathrm{~N}$ e a incerteza expandida de aproximadamente $6 \mathrm{~N}$. Para uma melhora significativa nestes valores, deve-se aumentar o número de corpos de prova para que o coeficiente de $t$-student seja melhorado. No caso prático em questão usou-se apenas 3 corpos de prova conforme a norma NBR 9176, sendo que para diminuir consideravelmente a incerteza dever-se-ia utilizar em torno de 20 unidades, porém o tempo e custo do ensaio aumentariam consideravelmente.

Outro ponto importante é relacionado à calibração das máquinas e instrumentos, pois operações e manipulação inadequada aumentam significativamente o valor do erro intrínseco dos valores de incerteza, isso se verifica na Tabela 3.4, onde aparecem os valores de incerteza expandida oriundos de Certificados de Calibração. 


\section{REFERÊNCIAS}

ASSOCIAÇÃO BRASILEIRA DE NORMAS TÉCNICAS (ABNT). ANEXO DA PORTARIA INMETRO N ${ }^{\circ}$ 079/2011: Requisitos de Avaliação da Conformidade (RAC) para Colchões e Colchonetes de Espuma Flexível de Poliuretano. Rio de Janeiro, 2011.

ASSOCIAÇÃO BRASILEIRA DE NORMAS TÉCNICAS (ABNT). Norma Brasileira NBR 9176: Espuma Flexível de Poliuretano - Determinação da Força de Indentação, Rio de Janeiro, 2003.

ASSOCIAÇÃO BRASILEIRA DE NORMAS TÉCNICAS (ABNT). Norma Brasileira NBR 9177: Espuma Flexível de Poliuretano - Determinação da Fadiga Dinâmica, Rio de Janeiro, 2003.

ASSOCIAÇÃO BRASILEIRA DE NORMAS TÉCNICAS (ABNT). Norma Brasileira NBR 13579-1: Colchão e Colchonete de Espuma Flexível de Poliuretano e Bases Parte 1: Requisitos e Métodos de Ensaio, 3. Ed, Rio de Janeiro, 2011.

ASSOCIAÇÃO BRASILEIRA DE NORMAS TÉCNICAS (ABNT). Norma Brasileira NBR 17025: Requisitos Gerais para a Competência de Laboratórios de Ensaios e Calibrações, Rio de Janeiro, 2005.

BENGTSSON, A. Uncertainty in Fatigue Life Prediction of Structures to Gussian Loads, Probabilistic Engineering Mechanics, v. 24, p. 224-235, Elsevier, 2009.

BUCAR, T. A Neural Network Approach to Describing the Satter of S-N Curves, International Journal of Fatigue, v. 28, p. 311-323, Elsevier, 2006.

CALLISTER, W. Materials Science and Engineering. 7 Ed. New York: John Wiley \& Sons Inc, 2007.

DIETER, G. Mechanical Metallurgy, 3 Ed. London: McGraw-Hill, 1988.

EUROPEAN CO-OPERATION FOR ACREDITATION, EA-4/16: Guidelines on the Expression of Uncertainty in Quantitative Testing, 2003.

INSTITUTO NACIONAL DE METROLOGIA, NORMALIZAÇÃO E QUALIDADE INDUSTRIAL (INMETRO), SOCIEDADE BRASILEIRA DE METROLOGIA (SBM) E ASSOCIAÇÃO BRASILEIRA DE NORMAS TÉCNICAS (ABNT), EA-4/02: Expressão da Incerteza de Medição na Calibração, 1 Ed. Rio de Janeiro, 1999.

INSTITUTO NACIONAL DE METROLOGIA, NORMALIZAÇÃO E QUALIDADE INDUSTRIAL (INMETRO), SOCIEDADE BRASILEIRA DE METROLOGIA (SBM) E ASSOCIAÇÃO BRASILEIRA DE NORMAS TÉCNICAS (ABNT), EA-4/02: Expressão da Incerteza de Medição na Calibração - Exemplos, 1 Ed. Rio de Janeiro, 1999. 
JOINT COMMITTEE FOR GUIDES IN METROLOGY (JCGM). JCGM 100: Evaluation of Measurement Data - Guide to the Expression of Uncertainty in Measurement (GUM), 2008a.

JOINT COMMITTEE FOR GUIDES IN METROLOGY (JCGM). JCGM 200: International Vocabulary of Metrology - Basic and General Concepts and Associated Terms (VIM) 2008b.

JORNADA, D. Implantação de um Guia Orientativo de Incerteza de Medição para Avaliadores de laboratório da Rede Metrológica RS, Dissertação PPGEP: UFRGS, 2009.

KARADENIZ, $\mathrm{H}$. Uncertainty Modeling in the Fatigue Reliability Calculation of Offshore Structures, Realiability Engineering \& System Safety, v. 74, p. 323-335, Elsevier, 2001.

KOUTSOURELAKIS, S. Effect of Material Uncertainties on Fatigue Life Calculations of Aircraft Fuselages: A Cohesive Element Model, Engineering Fracture Mechanics, v. 73, p. 1202-1219, Elsevier, 2006.

PIERCE, S. Uncertainty Analisys of a Neural Network Used for Fatigue Lifetime Prediction, Mechanical System and Signal Processing, v. 22, p.1395-1411, Elsevier, 2008.

PIZZOLATO, M. Mapeamento da Estrutura Global que Fornece Confiança às Medições: Análise da Inserção Brasileira, Tese PPGEP: UFRGS, 2006.

STANDARDS MEASUREMENT \& TEST MEASUREMENTS (SM\&T), UNCERTE CoP 05 - Manual of Codes of Practice for Determination of Uncertainties in Mechanical Tests on Metallic Material $n^{\circ} 05$ - The Determination of Uncertainties in Low Cycle Fatigue Testing, 2000.

SVENSSON, T. Prediction Uncertainties at Variable Amplitude Fatigue, International Journal of Fatigue, v. 19, p.295-302, Elsevier, 1997.

STEPHENS, R.; FATEMI, A.; STEPHENS, R. Metal Fatigue in Engineering, 2 Ed, New York: Wiley Interscience, 2001.

VILAR, W. Química e Tecnologia de Poliuretanos, 3 Ed, Rio de Janeiro, Vilar Consultoria, 2004. 